\title{
Calcium-Evoked Dendritic Exocytosis in Cultured Hippocampal Neurons. Part II: Mediation by Calcium/Calmodulin-Dependent Protein Kinase II
}

\author{
Mirjana Maletic-Savatic, Thillai Koothan, and Roberto Malinow \\ Cold Spring Harbor Laboratory, Cold Spring Harbor, New York 11724
}

Calcium-evoked dendritic exocytosis (CEDE), demonstrated in cultured hippocampal neurons, is a novel mechanism that could play a role in synaptic plasticity. A number of forms of neuronal plasticity are thought to be mediated by calcium/ calmodulin-dependent protein kinase II (CaMKII). Here, we investigate the role of CaMKII in CEDE. We find that the developmental time course of CEDE parallels the expression of $\alpha$ CaMKII, a dominant subunit of CaMKII. An inhibitor of this enzyme, KN-62, blocks CEDE. Furthermore, $7 \mathrm{~d}$ in vitro neurons (which normally do not express $\alpha$ CaMKII nor show CEDE) can undergo CEDE when infected with a recombinant virus produc- ing $\alpha$ CaMKII. Expression of a constitutively active CaMKII produces dendritic exocytosis in the absence of calcium stimulus, and this exocytosis is blocked by nocodazole, an inhibitor of microtubule polymerization that also blocks CEDE. These results indicate that CEDE is mediated by the activation of CaMKII, consistent with the view that CEDE plays a role in synaptic plasticity.

Key words: FM1-43; exocytosis; dendrites; pyramidal neurons; hippocampal cultures; CaMKII; KN-62; immunocytochemistry; vaccinia virus
Calcium-evoked dendritic exocytosis (CEDE) is a novel process that might be involved in the regulated delivery of dendritic and postsynaptic membrane-bound proteins. The essential feature of CEDE is its triggering by calcium. In principal projection neurons a major constituent of synapses is the calcium-sensitive enzyme calcium/calmodulin-dependent protein kinase II (CaMKII) (Erondu and Kennedy, 1985; Kennedy et al., 1990; Braun and Schulman, 1995; De Koninck and Schulman, 1998). In neurons, CaMKII is a large multimeric enzyme composed of $\alpha$ - and $\beta$-subunits (Bennett et al., 1983; Miller and Kennedy, 1985; Braun and Schulman, 1995). Very little of the $\alpha$-subunit is expressed early in development, but it dominates the composition of CaMKII as neurons mature (Scholz et al., 1988). The ability of CaMKII to autophosphorylate, thereby prolonging its actions, may enable this molecule to convert a transient signal to a long-lasting modification (Lisman, 1994). Indeed, several lines of evidence indicate that increased CaMKII activity mediates longterm potentiation (LTP), a form of activity-dependent synaptic plasticity that may underlie some forms of learning and memory. CaMKII activity is necessary for LTP, increases in CaMKII activity can be sufficient to generate potentiated transmission mimicking LTP, and LTP activates CaMKII (Malenka et al., 1989; Malinow et al., 1989; Silva et al., 1992; Fukunaga et al., 1993; Pettit et al., 1994; Lledo et al., 1995; Barria et al., 1997).

One model for LTP (Lynch and Baudry, 1984; Liao et al., 1993, 1995; Isaac et al., 1995) proposes the incorporation of glutamate receptors into postsynaptic sites. In the hippocampus, excitatory synaptic transmission is mediated by glutamate acting on both NMDA and AMPA receptors (Hestrin et al., 1990). NMDA

\footnotetext{
Received March 23, 1998; revised June 18, 1998; accepted June 22, 1998.

We thank the Mathers Foundation for generous support and Nancy Dawkins for preparing neuronal cultures.

Correspondence should be addressed to Dr. Roberto Malinow, Cold Spring Harbor Laboratory, Cold Spring Harbor, NY 11724.

Copyright (C) 1998 Society for Neuroscience $\quad 0270-6474 / 98 / 186814-\bullet \$ 05.00 / 0$
}

receptor contribution to synaptic transmission at resting membrane potential is sparse because of its voltage-dependent properties (Hestrin et al., 1990). In this LTP model some synapses have both AMPA and NMDA receptors, whereas other synapses have only NMDA receptors. These latter synapses are functionally silent, because at normal resting potential the transmitter release does not give any postsynaptic response. During LTP induction AMPA receptors could be inserted into the membrane of both kinds of synapses. This would transform silent synapses into functional ones and increase the response amplitude at those synapses that were functional before LTP. In this model the increase in AMPA receptors at synapses then could be responsible for the enhanced transmission. Replenishment of existing receptors, in the absence of LTP-inducing stimuli, could allow enhanced transmission to persist in the face of protein turnover.

However, little is known about the mechanisms that govern the targeting, clustering, and insertion of receptors into the postsynaptic membrane. At the best-characterized synapse, neuromuscular junction, acetylcholine receptor formation is an intrinsic property of the postsynaptic cell, whereas the recruitment of the receptor microclusters to form large postsynaptic aggregates is triggered by presynaptic events (Hall and Sanes, 1993). Hence, it is possible that in central neurons the receptors are confined in intracellular membranous compartments throughout a dendritic tree and are delivered selectively to postsynaptic sites where epigenetic factors like synaptic activity may induce their incorporation into the membrane. Recent electron microscopy studies on central excitatory synapses report the presence of intracellular membranous compartments and vesicles inside dendritic spines (Spacek and Harris, 1997) as well as intracellular AMPA receptors (Petralia and Wenthold, 1992; Rubio and Wenthold, 1997). Increased $\alpha \mathrm{CaMKII}$ selectively increases AMPA responses (Wu et al., 1996; Shirke and Malinow, 1997) and decreases silent synapses (Wu et al., 1996). Furthermore, a recent study indicated that LTP generation is blocked by inhibitors of regulated postsyn- 


\section{DIV}

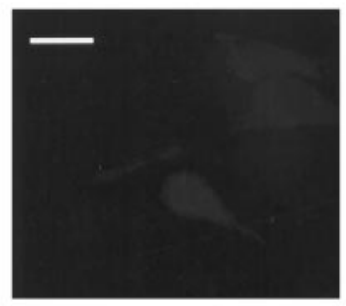

7 DIV

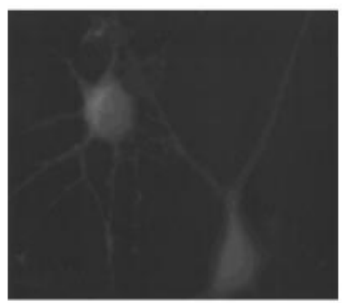

9 DIV

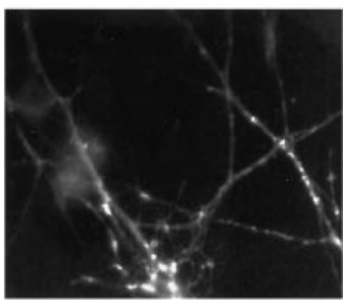

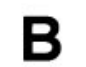

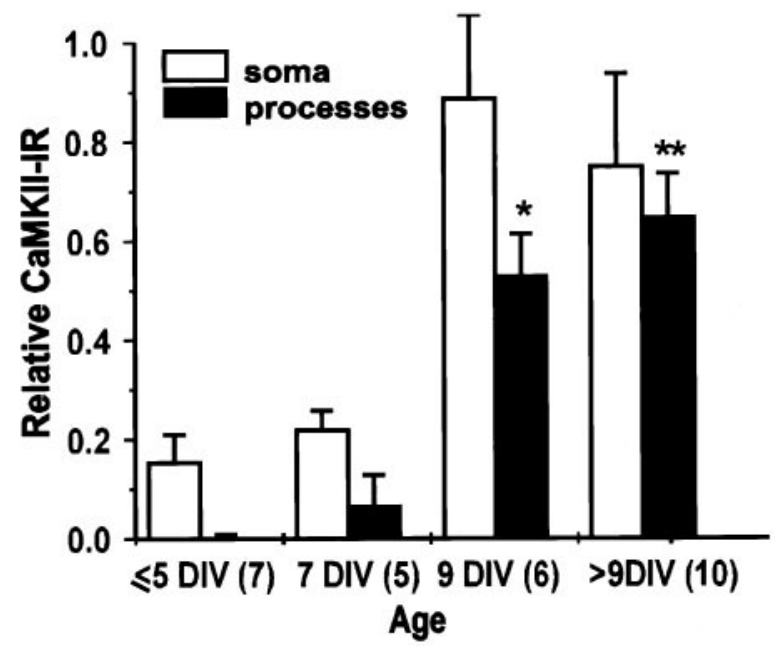

Figure 1. $\alpha \mathrm{CaMKII}$ expression correlates with CEDE. $A$ $\alpha$ CaMKII immunoreactivity (CaMKII-IR) in neurons 5 (left), 7 (middle), and 9 (right) DIV old. Scale bar, $20 \mu \mathrm{m}$. $B$, Plot of the CaMKII-IR in soma and processes of developing cultured neurons. $\alpha \mathrm{CaMKII-IR}$ in neuronal somata and processes was normalized by $\alpha$ CaMKII-IR in nearby astrocytes. Sample size is indicated in parentheses. ${ }^{*} p<0.05 ; * * p<0.01$. aptic exocytosis (Lledo et al., 1998). These studies suggest that CEDE could be one of the mechanisms by which intracellular AMPA receptors are delivered into synapses during plasticity. Here we investigate the role of CaMKII in CEDE. We find that CEDE, like LTP, also requires the presence and activity of CaMKII, and an increase in CaMKII activity triggers CEDE. Therefore, we report additional evidence that links regulated postsynaptic exocytosis and LTP.

\section{MATERIALS AND METHODS}

\section{Cell culture}

Cortical astrocytes were derived from 1-d-old rat pups and plated onto poly-L-lysine-coated coverslips. Hippocampal neurons were generated from 19-d-old rat embryos (Banker and Goslin, 1990) and plated onto a confluent monolayer of astrocytes. Astrocytes and neurons were plated at 50,000 and 30,000 cells per 18-mm-round glass coverslip (Fisher Scientific, Pittsburgh, PA), respectively. For relocation experiments the astrocytes and neurons were plated at 100,000 and 85,000 cells per 25-mm-square gridded glass coverslip (Bellco Glass, Vineland, NJ), respectively. Cultures were maintained in a serum-free medium (Banker and Goslin, 1990).

\section{Immunocytochemistry}

Cultures (3-15 d in vitro, DIV) were fixed in $4 \%$ paraformaldehyde in 0.1 M PBS containing $0.12 \mathrm{M}$ sucrose, for $20 \mathrm{~min}$ at $+4^{\circ} \mathrm{C}$, and immediately permeabilized with $0.3 \%$ Triton $\mathrm{X}-100$ in PBS for $5 \mathrm{~min}$ at room temperature. They were incubated in blocking solution $(10 \%$ horse serum, $1 \%$ goat-serum, and $0.1 \%$ Triton X-100 in PBS) for $1 \mathrm{hr}$ at room temperature and then immunostained overnight at $+4^{\circ} \mathrm{C}$ with the primary $\alpha$ CaMKII-specific monoclonal antibody $(10 \mu \mathrm{g} / \mathrm{ml}$; Boehringer Mannheim, Indianapolis, IN). After the washout of the primary antibody with blocking solution, the cultures were exposed to secondary FITCconjugated goat anti-mouse antibody $(30.8 \mu \mathrm{g} / \mathrm{ml}$; Cappel, West Chester, PA) for $2 \mathrm{hr}$ at room temperature. Immunoreactivity (IR) was analyzed immediately after the secondary antibody was washed away [FITC filter, Zeiss Axioskop (Oberkochen, Germany), $50 \mathrm{~W}$ mercury lamp].

\section{Labeling neurons with FM1-43}

Cultures were exposed for $16-36 \mathrm{hr}$ to $1.5 \mu \mathrm{M}$ FM1-43 at $35.5^{\circ} \mathrm{C}$ before being transferred to the microscope stage. Cultures were rinsed with $0.1 \%$ DMSO for 1 min and maintained in constantly perfusing bath solution for $1 \mathrm{hr}$. The bath solution [containing (in $\mathrm{mm}$ ) $119 \mathrm{NaCl}, 2.5$ $\mathrm{KCl}, 1.3 \mathrm{MgCl}_{2}, 2.5 \mathrm{CaCl}_{2}, 1 \mathrm{NaH}_{2} \mathrm{PO}_{4}, 26.2 \mathrm{NaHCO}_{3}$, and 11 glucose] also contained $1 \mu \mathrm{M}$ tetrodotoxin to abolish action potentials.

\section{Calcium-evoked dendritic exocytosis}

After overnight loading with $1.5 \mu \mathrm{M}$ FM1-43, the cultures were transferred to the microscope stage and maintained in constantly perfusing bath solution for $1 \mathrm{hr}$. Calcium ionophore A23187 (1 $\mu \mathrm{M})$ was applied directly onto the coverslip for $1 \mathrm{~min}$. Fluorescence was monitored for 30 min before and 30 min after A23187 application, at 15 min intervals. An additional image was taken $\sim 5 \mathrm{~min}$ after ionophore application. All 
A

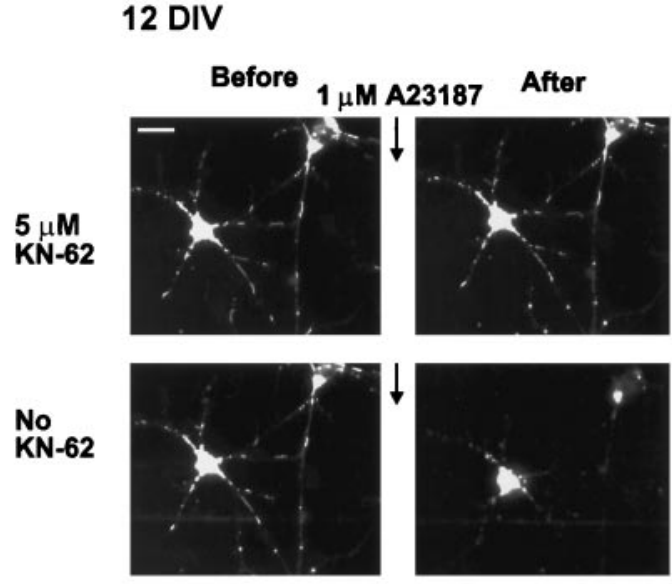

B

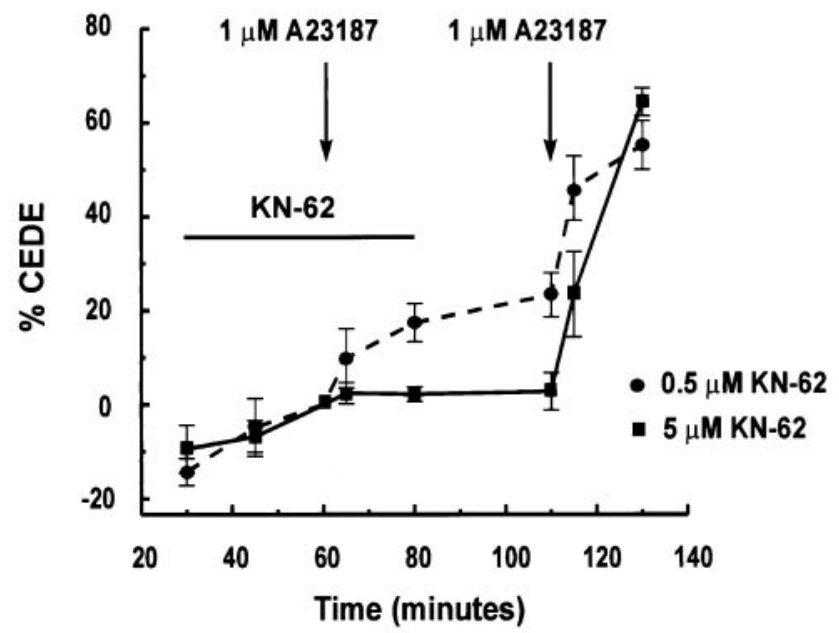

Figure 2. CaMKII activity is necessary for CEDE. A, KN-62 $(5 \mu \mathrm{M})$ prevents CEDE. The 12 DIV culture, loaded overnight with FM1-43, was challenged with A23187 in the presence (top two panels) and after the washout (bottom two panels) of $5 \mu \mathrm{M} \mathrm{KN}-62$, a CaMKII inhibitor. Scale bar, $20 \mu \mathrm{m}$. B. Plot of FM1-43 fluorescence observed in overnight-loaded mature neurons ( $>9$ DIV) transiently challenged with A23187 (indicated by arrows) in the presence of $0.5 \mu \mathrm{M}$ (dotted line; $n=3$ ) or $5 \mu \mathrm{M} \mathrm{KN}-62$ (solid line; $n=8 ;{ }^{*} p<0.05$ ) and after the subsequent washout of the drug.

images in a given experiment were taken with the same exposure time (100-500 msec, chosen so that the fluorescent signal did not saturate the camera detection limit).

$K N-62$ treatment. Mature cultures ( $>9$ DIV) were loaded overnight with $1.5 \mu \mathrm{M}$ FM1-43 and then exposed to $0.5 \mu \mathrm{M}$ or $5 \mu \mathrm{M} \mathrm{KN}-62$ for 1.5 $\mathrm{hr}$ (during the $1 \mathrm{hr}$ initial wash period and 30 min after A23187 application). KN-62-containing bath solution was replaced with fresh bath solution. Thirty minutes later A23187 was reapplied, and its effect was monitored for $20 \mathrm{~min}$.

\section{Infections}

Vaccinia virus was constructed and purified as described (Pettit et al., $1995)$. Viruses contain a strong synthetic promoter driving the expression of either $\beta$-galactosidase alone $(\beta \mathrm{GALVV})$ or the protein of interest$\alpha$ CaMKII (fCaMKIIVV) or $\alpha$ CaMKII(1-290) (tCaMKIIVV). In the viruses expressing CaMKII, a second ( $\sim 40$ times weaker) promoter drives the expression of $\beta$-galactosidase. Cultures were infected at 3-5 multiplicity of infection (MOIs) for $6 \mathrm{hr}$ at $35.5^{\circ} \mathrm{C}$ after a $12 \mathrm{hr}$ exposure to FM1-43 (fCaMKIIVV, total exposure to FM1-43 was $18 \mathrm{hr}$ ) or at 5-10 MOIs for $12 \mathrm{hr}$ at $35.5^{\circ} \mathrm{C}$, along with the FM1-43 (tCaMKIIVV). More than $90 \%$ infection was confirmed by X-gal staining in $\beta G A L V V-$, fCaMKIIVV-, and tCaMKIIVV-infected cells.
Nocodazole treatment. Mature cultures ( $>9$ DIV), loaded overnight with $1.5 \mu \mathrm{M}$ FM1-43 and infected with the tCaMKIIVV, were exposed for $45 \mathrm{~min}$ to $2.5 \mu \mathrm{g} / \mathrm{ml}$ nocodazole in the presence of $1.5 \mu \mathrm{M}$ FM1-43 at $35.5^{\circ} \mathrm{C}$. Experiments were performed as above.

\section{Microscopy}

Images were acquired with a computer-controlled cooled CCD camera (Photometrics, Tucson, AZ), using FITC filters (peak at $450 \mathrm{~nm}$ ) on a Zeiss Axioskop epifluorescence microscope (50 W mercury lamp), and processed with Photometrics-supplied software (PMIS).

\section{Data analysis}

For quantitative measurements of CEDE, several regions of interest (2-5) were placed on dendrites (locations chosen before post-treatment images were analyzed), and FM1-43 fluorescence was integrated. Background fluorescence, measured in regions next to the neurons, was subtracted. FM1-43 fluorescence values were normalized to the intensity observed immediately before A23187 application. Statistical analysis was done by one-way ANOVA.

\section{RESULTS}

Our studies of CEDE (Maletic-Savatic and Malinow, 1998) indicate that this process is regulated developmentally, because we observed it only in mature cultured hippocampal neurons. We sought to identify which factor or factors made neurons 9 DIV or older competent to show CEDE. We concentrated our efforts on one $\mathrm{Ca}^{2+}$-activated enzyme, CaMKII (Braun and Schulman, 1995), which previously has been demonstrated to show developmentally regulated expression (Kelly and Vernon, 1985). Using immunocytochemistry, we found that a major constituent of CaMKII, $\alpha$ CaMKII, had a similar developmental expression pattern to the onset of CEDE. In particular, in our culture conditions the expression level of $\alpha \mathrm{CaMKII}$ in processes was very weak at 7 DIV and robust by 9 DIV (Fig. $1 A$ ). In addition, at 7 DIV $\alpha$ CaMKII was detected only as a homogeneous perinuclear staining, whereas from 9 DIV onward it accumulated as distinct, intensely stained patches in dendrites. As previously reported (Scholz et al., 1988; Tighilet et al., 1998), the enzyme was not expressed in astrocytes, which form a supportive confluent monolayer of cells beneath hippocampal neurons. We performed a quantitative analysis of the $\alpha \mathrm{CaMKII}$ expression in neuronal soma and processes (Fig. $1 B$ ). $\alpha$ CaMKII-IR in processes increased with the age of cultures, with a particularly large rise from 7 to 9 DIV. Similarly, we observed CEDE only in neurons older than 9 DIV (Maletic-Savatic and Malinow, 1998).

To determine whether CaMKII activation is necessary for CEDE, we tested the effect of KN-62, an inhibitor of CaMKII (Tokumitsu et al., 1990). Mature neurons were loaded overnight with FM1-43 and treated for $1 \mathrm{hr}$ with 0.5 or $5 \mu \mathrm{M} \mathrm{KN}-62$. Calcium ionophore challenge produced no significant CEDE in the presence of $5 \mu \mathrm{M} \mathrm{KN}-62(3.1 \pm 1.5 \%$ of control CEDE, $n=$ 8; Fig. $2 A, B)$ and $31.6 \pm 6.8 \%$ of control CEDE in the presence of $0.5 \mu \mathrm{M} \mathrm{KN}-62(n=3$; Fig. $2 B)$. On washout of the inhibitor, a subsequent calcium ionophore challenge produced robust exocytosis (Fig. 2). These results indicate that CEDE requires CaMKII activity.

Is diminished CaMKII activity, as a consequence of the lack of $\alpha \mathrm{CaMKII}$, the critical component lacking in 7 DIV neurons, making them incompetent to display CEDE? We directly tested this hypothesis by introducing $\alpha \mathrm{CaMKII}$ into neurons, using a recombinant vaccinia virus expression system (Pettit et al., 1994, 1995) at an early developmental age when endogenous $\alpha$ CaMKII was not present. We determined conditions that allowed for the infection and expression of recombinant product in virtually all of the cells in a culture dish (Fig. $3 A$ ). We visualized the expression 

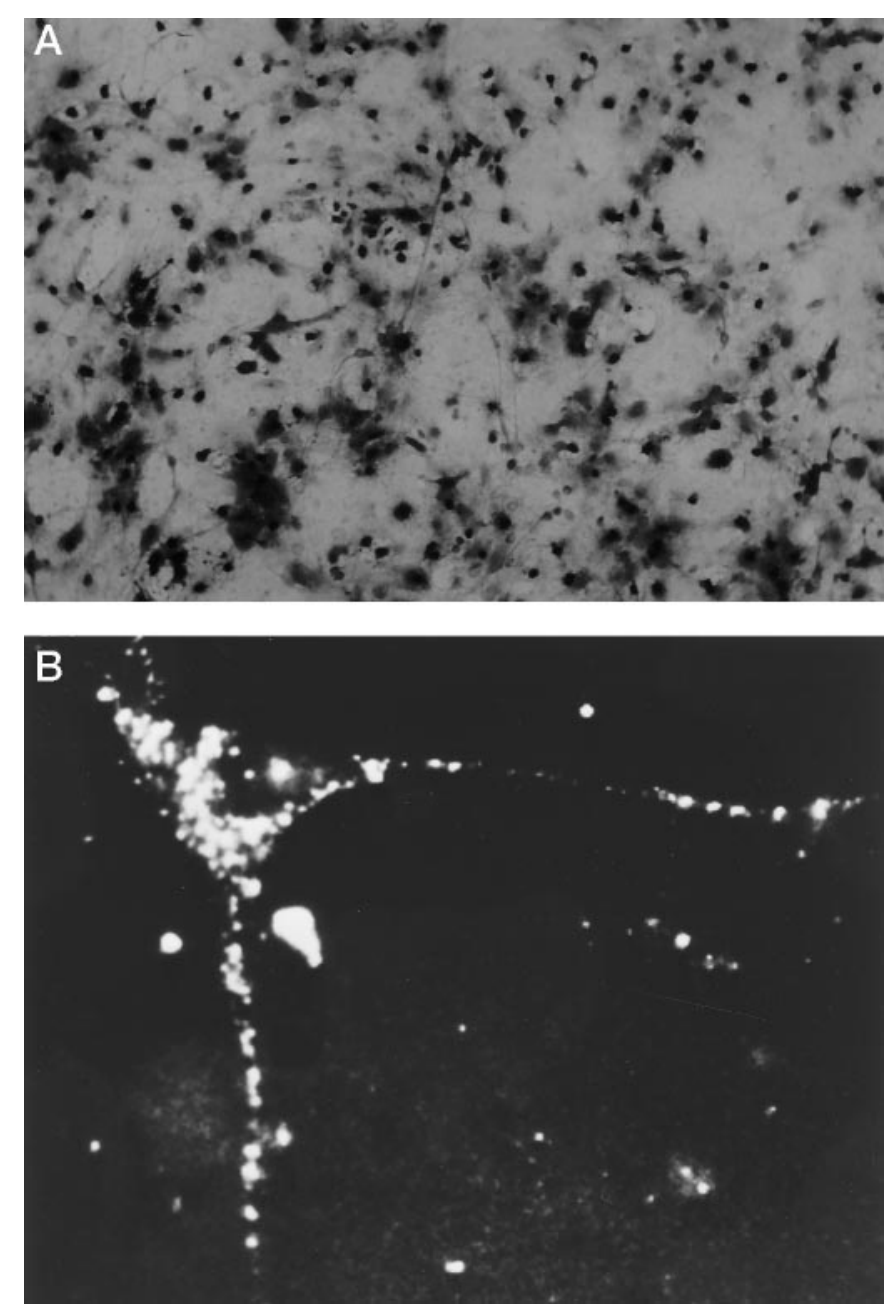

Figure 3. Infection of cultured neurons with the vaccinia virus. $A$, A large fraction $(>90 \%)$ of cells in cultures exposed to a vaccinia virus (VV) producing recombinant $\beta$-galactosidase $(\beta \mathrm{GALVV})$ expresses recombinant $\beta$-galactosidase, indicated by X-GAL staining. $B$, Expression of recombinant $\alpha \mathrm{CaMKII}$ in a neuron infected with a VV producing both recombinant hemagglutinin epitope-tagged $\alpha \mathrm{CaMKII}$ and $\beta$-galactosidase (fCaMKIIVV), indicated by punctate immunofluorescent staining with the use of a hemagglutinin epitope antibody.

pattern of a recombinant hemagglutinin epitope-tagged $\alpha \mathrm{CaMKII}$ within a neuron (Fig. $3 B$ ). Virally expressed recombinant $\alpha \mathrm{CaMKII}$ was delivered to dendrites and showed a punctate staining pattern, similar to that seen for endogenous $\alpha \mathrm{CaMKII}$ (see Fig. 1A).

As shown in Figure $4 A, 7$ DIV neurons with no infection or infected with a virus producing only the control protein $\beta$-galactosidase showed no CEDE. However, 7 DIV neurons infected with a virus producing $\alpha$ CaMKII (fCaMKII; Figs. $3 B$, $4 A$ ) after overnight FM1-43 loading showed CEDE that was comparable in magnitude to that seen with 9 DIV neurons in control conditions (Fig. 4A). Interestingly, 9 DIV neurons infected with a virus producing $\alpha$ CaMKII showed larger CEDE than control 9 DIV neurons (Fig. 4B), suggesting that the amount of $\alpha \mathrm{CaMKII}$ protein is rate-limiting for CEDE in these neurons. Also, neurons $<7$ DIV showed little CEDE when infected with a virus-producing $\alpha$ CaMKII (Fig. 4B), suggesting that developmentally regulated factors other than $\alpha$ CaMKII also control CEDE.
To test if the activity of CaMKII is sufficient to produce dendritic exocytosis, we analyzed the effect of a truncated, constitutively active form of CaMKII (tCaMKII) that contains only the catalytic domain of the enzyme (Pettit et al., 1994). The infection of neurons with a recombinant vaccinia virus producing tCaMKII (tCaMKIIVV) has been shown previously to increase CaMKII activity (Pettit et al., 1994). We loaded mature neurons overnight with FM1-43 and infected them for $12 \mathrm{hr}$ with tCaMKIIVV. Neurons that were not infected or infected with a virus producing the control protein ( $\beta$-galactosidase) showed little loss of dye during a $1 \mathrm{hr}$ observation period (Fig. 5). However, neurons infected with tCaMKIIVV showed a significant loss of FM1-43 (Fig. 5). This indicates that constitutively active CaMKII can induce dendritic exocytosis in the absence of a calcium stimulus.

To determine possible downstream targets of CaMKII mediating dendritic exocytosis, we investigated the effects of nocodazole, an agent producing microtubule depolymerization (Goslin et al., 1989). Nocodazole effectively blocked CEDE (MaleticSavatic and Malinow, 1998). In addition, nocodazole blocked the exocytosis produced by tCaMKIIVV (Fig. 5), indicating that intact microtubules are required for dendritic exocytosis.

\section{DISCUSSION}

\section{CaMKII activity and CEDE}

The principal findings of this study are that the activation of CaMKII is both necessary and can be sufficient to generate CEDE. Here we report four independent experimental approaches that support these findings: (1) the expression of $\alpha$ CaMKII, a major constituent of CaMKII, in dendritic processes of cultured hippocampal neurons parallels the developmental time course of CEDE; (2) an inhibitor of CaMKII, $\mathrm{KN}-62$, prevents CEDE; (3) neurons 7 DIV that normally neither express $\alpha \mathrm{CaMKII}$ nor show CEDE become competent to manifest $\mathrm{CEDE}$ when infected with a recombinant virus producing $\alpha \mathrm{CaMKII}$; and (4) neurons infected with a recombinant virus producing constitutively active CaMKII show dendritic exocytosis in the absence of a calcium stimulus. These results indicate that CaMKII activation mediates CEDE in these neurons. Therefore, these results further characterize CEDE and assign another function to CaMKII.

Our studies of $\alpha \mathrm{CaMKII}$ immunoreactivity generally agree with earlier studies on the developmental expression of this subunit of CaMKII in cultured hippocampal neurons (Scholz et al., 1988). Little $\alpha$ CaMKII immunoreactivity is seen before 9 DIV, at which point there is a fairly abrupt increase in expression, particularly in processes. A similar developmental increase was reported for the phosphorylation of synaptic junction proteins by endogenous CaMKII (Kelly et al., 1987). In our cultures, synaptic responses appear $\sim 7 \mathrm{DIV}$, becoming much more pronounced over the next few days (our unpublished results). It appears that, with such an expression pattern, $\alpha$ CaMKII is well poised to participate in synapse formation or maturation. Indeed, such a role has been proposed for CaMKII in the maturation of retinotectal synapses (Wu et al., 1996).

Our studies of CaMKII expression indicate that this enzyme, or at least the $\alpha$-subunit, dramatically increases developmentally at the same time as that when we detect CEDE. However, is the activity of CaMKII necessary for CEDE? To test this hypothesis, we blocked CaMKII activity with KN-62 (see Fig. 2). These experiments showed a concentration-dependent block of CEDE by KN-62. Because this drug also blocks CaMKIV, with this 
A

\section{DIV}
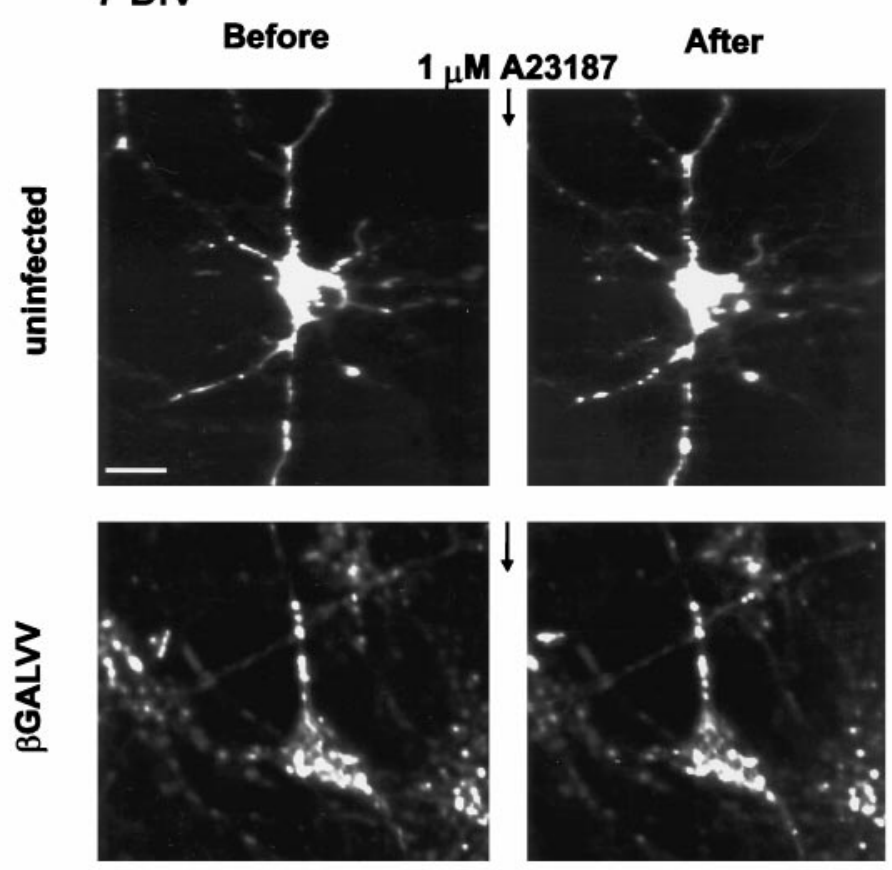

B
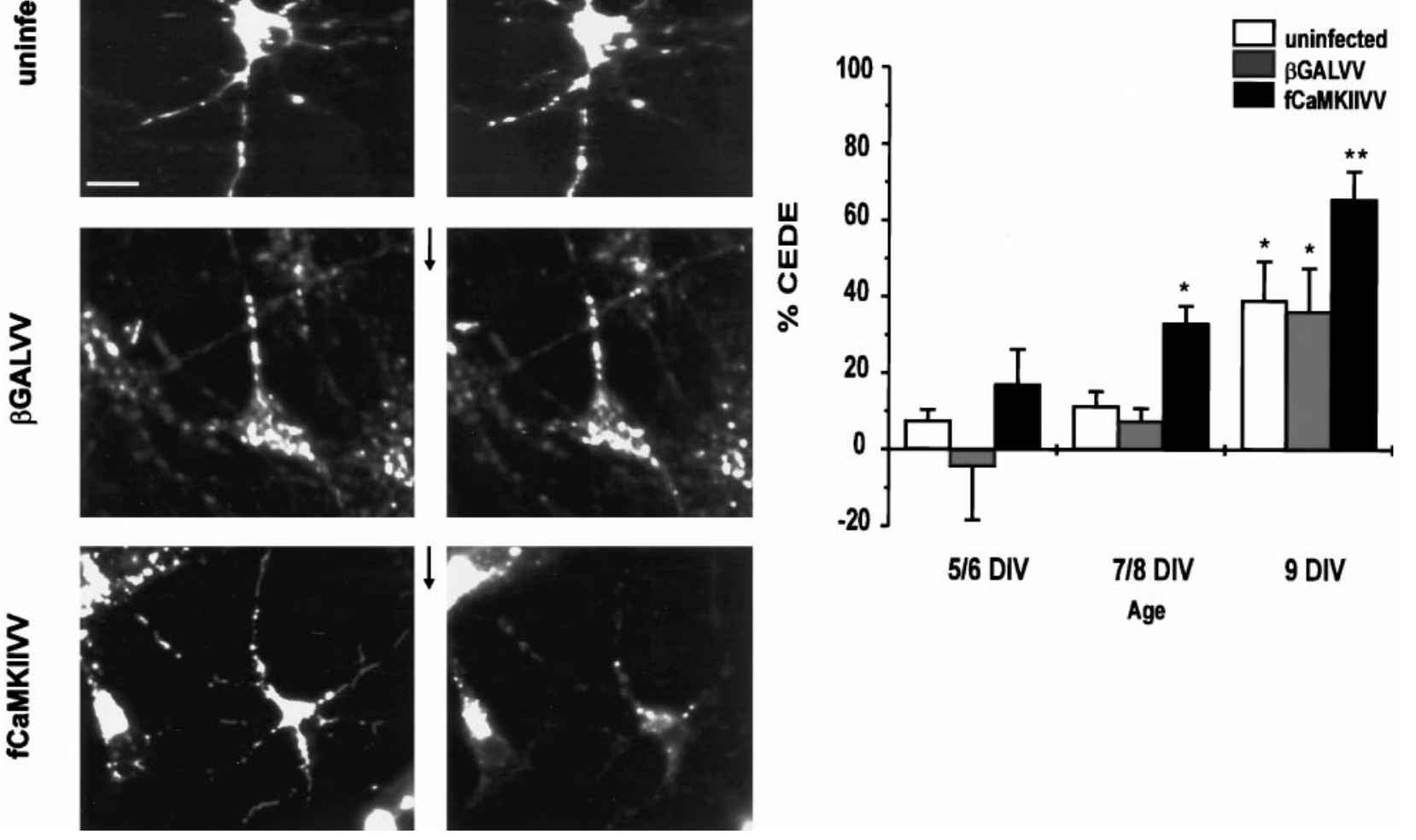

Figure 4. Recombinant $\alpha$ CaMKII renders immature neurons competent to show CEDE. $A$, Neurons (7 DIV) loaded overnight with FM1-43 were uninfected (top) or were infected with $\beta$ GALVV (middle) or CaMKIIVV (bottom). A23187 (1 $\mu \mathrm{M}$ for 1 min) evoked no significant exocytosis in uninfected and $\beta$ GALVV-infected neurons but produced significant exocytosis in CaMKIIVV-infected neurons. Scale bar, $10 \mu \mathrm{m}$. $B$, Quantitative analysis of CEDE in control and infected developing neurons (age is indicated). The following number of cultures was analyzed for each experiment: $5 / 6$ DIV, control $n=12, \beta$ GALVV $n=7$, CaMKIIVV $n=8 ; 7 / 8$ DIV, control $n=15, \beta$ GALVV $n=8$, CaMKIIVV $n=9 ; 9 D I V$, control $n=8$, $\beta$ GALVV $n=5$, CaMKIIVV $n=5$. * $p<0.05$; ${ }^{*} p<0.01$. At no age was CEDE significantly different in $\beta$ GALVV and uninfected neurons.

experiment we cannot rule out the possibility that this enzyme is required for CEDE.

Possibly the most compelling results indicating a role for CaMKII in CEDE come from the experiments in which recombinant $\alpha \mathrm{CaMKII}$ is expressed in 7 DIV neurons. At this point in development these neurons normally neither express $\alpha \mathrm{CaMKII}$ nor show CEDE. However, the expression of recombinant $\alpha \mathrm{CaMKII}$ renders these neurons competent to show CEDE. The expression of constitutively active CaMKII demonstrated that activity of this enzyme, in the absence of a calcium stimulus, is sufficient to trigger dendritic exocytosis.

CaMKII has been implicated in the regulation of presynaptic exocytosis, acting on actin binding of presynaptic vesicles (Ceccaldi et al., 1995). CaMKII injected presynaptically in squid giant synapse facilitated transmitter release (Llinás et al., 1991). Additionally, the extracellular application of synthetic peptide inhibitors of CaMKII suppressed the phosphorylation of synapsin I at the CaMKII-specific site and decreased excitatory synaptic responses elicited in the CA1 hippocampal region (Waxham et al., 1993). On the basis of these results CaMKII is postulated to play an essential role in the recruitment of presynaptic vesicles, pos- sibly by freeing vesicles from an actin-bound pool. In addition, CaMKII has been implicated in the exocytosis of dense-core vesicles in PC12 cells (Schweitzer et al., 1995). These experiments indicated that CaMKII action modulates a step between $\mathrm{Ca}^{2+}$ influx and the fusion of dense-core vesicles in PC12 cells. In addition to its role in presynaptic function, CaMKII has been implicated in mediating cell membrane resealing via a vesicular exocytotic process (Steinhardt et al., 1994).

Our results indicate that CaMKII acts also as a regulator of dendritic and postsynaptic exocytosis. What are the downstream targets of active CaMKII in dendrites? We find that depolymerization of microtubules prevents exocytosis produced by constitutive CaMKII activity, indicating that microtubule function is required in parallel or downstream of CaMKII activity to produce CEDE. CEDE is a process that involves exocytosis of smooth endoplasmic reticulum/trans-Golgi network (SER/ TGN)-like organelles in dendrites (Maletic-Savatic and Malinow, 1998). TGN in cultured neurons is associated with microtubules, because treatment with nocodazole induces the scattering of its vesicles (Lowenstein et al., 1994). One of the possible mechanisms of CaMKII action in CEDE is the phosphorylation of 


\section{A 14 DIV}

0
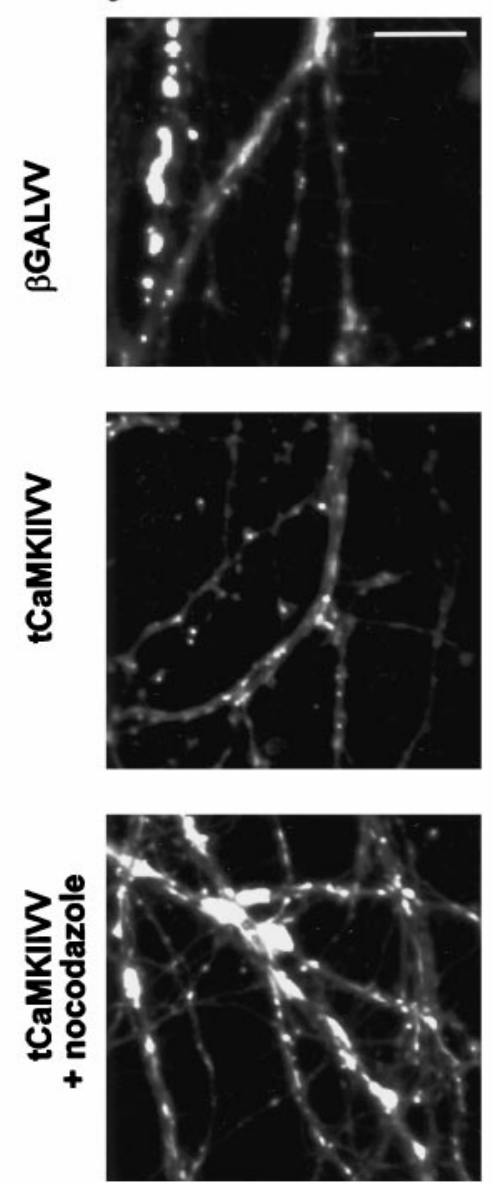

1
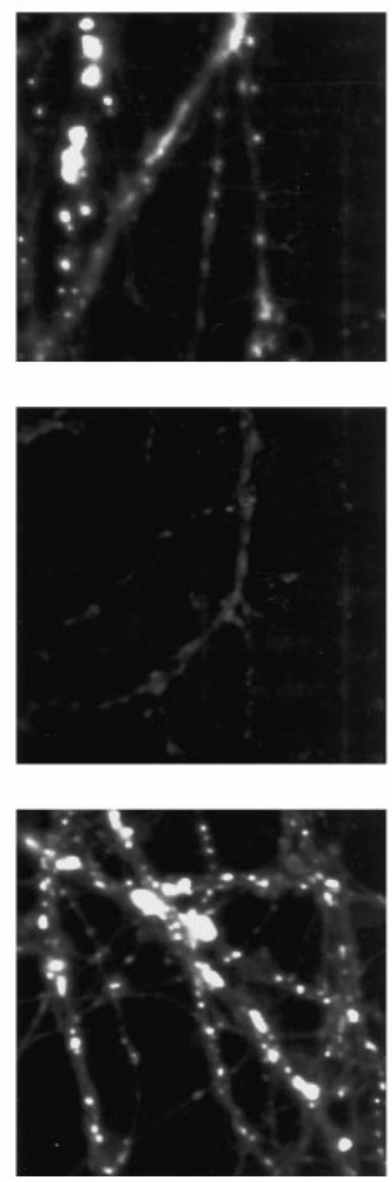

B

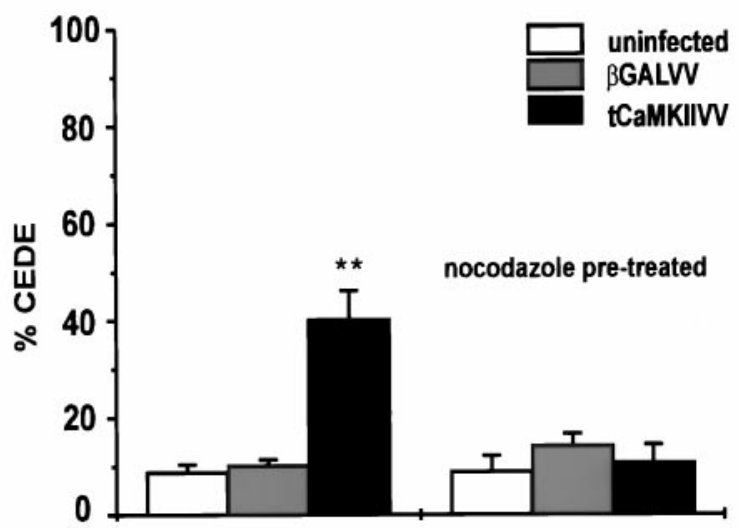

(4) (4) (5)
(4) (5) (6)

Figure 5. Recombinant $\alpha$ CaMKII can be sufficient to produce dendritic exocytosis. $A$, Constitutively active $\alpha$ CaMKII (tCaMKII) produces dendritic exocytosis in the absence of a calcium stimulus and is blocked by nocodazole. Fluorescence images were obtained at time 0 (after $1 \mathrm{hr}$ of wash, left panels) and $1 \mathrm{hr}$ later (right panels) of cultures infected with $\beta$ GALVV (top), tCaMKIIVV (middle), or tCaMKIIVV with nocodazole treatment (bottom). Scale bar, $10 \mu \mathrm{m}$. B. Fractional loss of fluorescence (indicating dendritic exocytosis) observed over $1 \mathrm{hr}$ for the conditions indicated. Sample size in parentheses refers to the number of cultures examined. ${ }^{*} p<0.01$.

microtubule-associated proteins (MAPs), which are known to be CaMKII substrates (Braun and Schulman, 1995). Phosphorylation of MAPs by CaMKII has been proposed as the mechanism of dendritic extension (Diez-Guerra and Avila, 1995), and this process could involve exocytosis like CEDE. Thus, microtubules could act as docking sites for vesicles and proteins, some of which would be CaMKII targets. Alternatively, microtubules could be necessary to deliver vesicles to exocytotic sites, at which point CaMKII could facilitate exocytosis by phosphorylating constituents of the exocytotic machinery.

\section{CEDE and LTP}

A number of studies indicate that postsynaptic CaMKII activity is necessary and sufficient for LTP, a form of activity-dependent synaptic plasticity observed in hippocampal neurons (Malenka et al., 1989; Malinow et al., 1989; Silva et al., 1992; Pettit et al., 1994; Lledo et al., 1995). Could CEDE play a role in LTP? It has been reported recently that postsynaptic exocytosis is required for LTP (Lledo et al., 1998). During LTP induction localized calcium entry might trigger the insertion of glutamate receptors (stored in intracellular compartments or perisynaptic regions; Hampson et al., 1992; Baude et al., 1995) into synapses by a CEDE-like process. This would change the postsynaptic site functionally so that it would produce larger responses to synaptically released transmitter. Interestingly, a recent study found that, during LTP induction, CaMKII activation and AMPA receptor phosphorylation (Barria et al., 1997) occur with a time course that closely parallels the time course we have found for CEDE (MaleticSavatic and Malinow, 1998). We have shown that CEDE is associated spatially with the spine-like structures in cultured hippocampal neurons and that it involves the exocytosis of SER/ TGN-derived organelles (Maletic-Savatic and Malinow, 1998). With SER and smooth vesicles present in the spine apparatus (Spacek and Harris, 1997) and CaMKII, an abundant protein in the postsynaptic density (Kennedy et al., 1983), all of the machinery necessary for CEDE is likely to be present in a spine, the site where synaptic plasticity occurs.

Although our studies did not demonstrate the relation between CEDE and synaptic plasticity directly, our data are quite suggestive that these phenomena might be associated. It is well established that increases in intracellular levels of $\mathrm{Ca}^{2+}$ at synaptic 


\section{A Insertion of receptors}

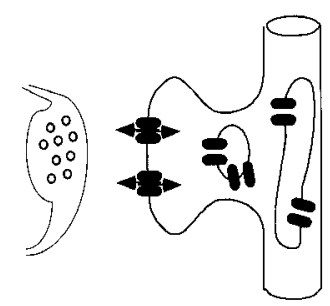

B Exocytosis into synaptic cleft
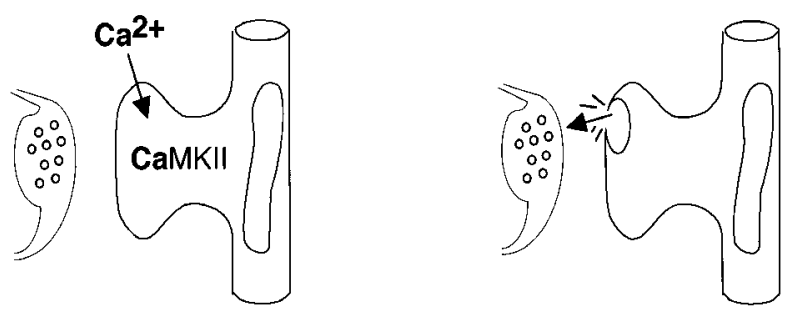

\section{Growth of the spine}

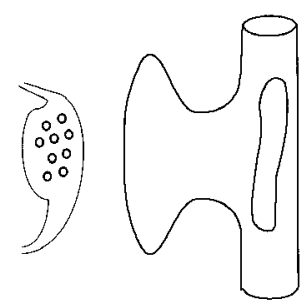

Figure 6. $\mathrm{CEDE}$ as a possible mechanism of synaptic plasticity. $\mathrm{Ca}^{2+}$ induced activation of $\alpha \mathrm{CaMKII}$ leads to the regulated fusion of perisynaptic SER/TGN-derived organelles with the dendritic plasma membrane. This fusion could $(A)$ expose new receptors at the surface of the postsynaptic cell, $(B)$ release factors that might affect presynaptic function, or $(C)$ provide more membrane for the growth of synapses.

regions as well as the activation of CaMKII play central roles in triggering LTP. Similarly, $\mathrm{Ca}^{2+}$ elevations and CaMKII activity trigger and mediate CEDE of organelles close to synapses. Therefore, these findings are consistent with the view that CEDE plays a role in LTP.

A variety of model mechanisms has been proposed to explain the cellular changes that underlie synaptic plasticity. Some models suggest that an increase in synaptic strength can occur without any obvious change in synaptic structure, whereas others propose that synaptic plasticity is associated with the structural change at the synapse. On the basis of our results, we propose the following model of synaptic plasticity mechanism that might change the postsynaptic site both functionally and structurally (Fig. 6). SER/ TGN-like organelles that are present in dendrites and perisynaptic sites are attached to microtubules. During repetitive synaptic transmission $\mathrm{Ca}^{2+}$ enters through NMDA receptors and activates CaMKII. Possibly by phosphorylation of some MAPs, this leads to regulated fusion of SER/TGN-derived organelles with the dendritic plasma membrane. This fusion might expose new receptors at the surface of the postsynaptic cell, release factors that might affect presynaptic function, or provide more membrane for the growth of the synapses.

\section{REFERENCES}

Banker GA, Goslin K (1990) Culturing nerve cells. Cambridge, MA: MIT.
Barria A, Muller D, Derkach V, Griffith LC, Soderling TR (1997) Regulatory phosphorylation of AMPA-type glutamate receptors by CaMKII during long-term potentiation. Science 276:2042-2045.

Baude A, Nusser Z, Molnar E, McIlhinney RA, Somogyi P (1995) High-resolution immunogold localization of AMPA-type glutamate receptor subunits at synaptic and non-synaptic sites in rat hippocampus. Neuroscience 69:1031-1055.

Bennett MK, Erondu NE, Kennedy MB (1983) Purification and characterization of a calmodulin-dependent protein kinase that is highly concentrated in brain. J Biol Chem 258:12735-12744.

Braun AP, Schulman H (1995) The multifunctional calcium/ calmodulin-dependent protein kinase: from form to function. Annu Rev Physiol 57:417-445.

Ceccaldi PE, Grohovaz F, Benfenati F, Chieregatti E, Greengard P, Valtorta F (1995) Dephosphorylated synapsin I anchors synaptic vesicles to actin cytoskeleton: an analysis by videomicroscopy. J Cell Biol 128:905-912.

De Koninck P, Schulman H (1998) Sensitivity of CaM kinase II to the frequency of $\mathrm{Ca}^{2+}$ oscillations. Science 279:227-230.

Diez-Guerra FJ, Avila J (1995) An increase in phosphorylation of microtubule-associated protein 2 accompanies dendrite extension during the differentiation of cultured hippocampal neurones. Eur J Biochem 227:68-77.

Erondu NE, Kennedy MB (1985) Regional distribution of type II Ca ${ }^{2+}$ / calmodulin-dependent protein kinase in rat brain. J Neurosci 5:3270-3277.

Fukunaga K, Stoppini L, Miyamoto E, Muller D (1993) Long-term potentiation is associated with an increased activity of $\mathrm{Ca}^{2+} /$ calmodulin-dependent protein kinase II. J Biol Chem 268:7863-7867.

Goslin K, Birgbauer E, Banker G, Solomon F (1989) The role of cytoskeleton in organizing growth cones: a microfilament-associated growth cone component depends upon microtubules for its localization. J Cell Biol 109:1621-1631.

Hall ZW, Sanes JR (1993) Synaptic structure and development: the neuromuscular junction. Cell [Suppl] 72:99-121.

Hampson DR, Huang XP, Oberdorfer M, Goh JW, Auyeung A, Wenthold RJ (1992) Localization of AMPA receptors in the hippocampus and cerebellum of the rat using an anti-receptor monoclonal antibody. Neuroscience 50:11-22.

Hestrin S, Nicoll RA, Perkel DJ, Sah P (1990) Analysis of excitatory synaptic action in pyramidal cells using whole-cell recording from rat hippocampal slices. J Physiol (Lond) 422:203-225.

Isaac JT, Nicoll RA, Malenka RC (1995) Evidence for silent synapses: implications for the expression of LTP. Neuron 15:427-434.

Kelly PT, Vernon P (1985) Changes in the subcellular distribution of calmodulin-kinase II during brain development. Brain Res 350:211-224.

Kelly PT, Shields S, Conway K, Yip R, Burgin K (1987) Developmental changes in calmodulin-kinase II activity at brain synaptic junctions: alterations in holoenzyme composition. J Neurochem 49:1927-1940.

Kennedy MB, Bennett MK, Erondu NE (1983) Biochemical and immunochemical evidence that the "major postsynaptic density protein" is a subunit of a calmodulin-dependent protein kinase. Proc Natl Acad Sci USA 80:7357-7361.

Kennedy MB, Bennett MK, Bulleit RF, Erondu NE, Jennings VR, Miller SG, Molloy SS, Patton BL, Schenker LJ (1990) Structure and regulation of type II calcium/calmodulin-dependent protein kinase in central nervous system neurons. Cold Spring Harb Symp Quant Biol 55:101110.

Liao D, Jones A, Malinow R (1993) Direct measurement of quantal changes underlying long-term potentiation in CA1 hippocampus. Neuron 9:1089-1097.

Liao D, Hessler NA, Malinow R (1995) Activation of postsynaptically silent synapses during pairing-induced LTP in CA1 region of hippocampal slice. Nature 375:400-404.

Lisman J (1994) The CaM kinase II hypothesis for the storage of synaptic memory. Trends Neurosci 17:406-412.

Lledo PM, Hjelmstad GO, Mukherji S, Soderling TR, Malenka RC, Nicoll RA (1995) Calcium/calmodulin-dependent kinase II and longterm potentiation enhance synaptic transmission by the same mechanism. Proc Natl Acad Sci USA 92:11175-11179.

Lledo PM, Zhang X, Sudhof TC, Malenka RC, Nicoll RA (1998) Postsynaptic membrane fusion and long-term potentiation. Science 279:399-403. 
Llinás R, Gruner JA, Sugimori M, McGuinness TL, Greengard P (1991) Regulation by synapsin I and $\mathrm{Ca}^{2+}$-calmodulin-dependent protein kinase II of the transmitter release in squid giant synapse. J Physiol (Lond) 436:257-282.

Lowenstein PR, Morrison EE, Bain D, Shering AF, Banting G, Douglas P, Castro MG (1994) Polarized distribution of the trans-Golgi network marker TGN38 during the in vitro development of neocortical neurons: effects of nocodazole and brefeldin-A. Eur J Neurosci 6:1453-1465.

Lynch G, Baudry M (1984) The biochemistry of memory: a new and specific hypothesis. Science 224:1057-1063.

Malenka RC, Kauer JA, Perkel DJ, Mauk MD, Kelly PT, Nicoll RA, Waxham MN (1989) An essential role for postsynaptic calmodulin and protein kinase activity in long-term potentiation. Nature 340:554-557.

Maletic-Savatic M, Malinow R (1998) Calcium-evoked dendritic exocytosis in cultured hippocampal neurons. Part I: trans-Golgi networkderived organelles undergo regulated exocytosis. J Neurosci 18:6803-6813.

Malinow R, Schulman H, Tsien RW (1989) Inhibition of postsynaptic PKC or CaMKII blocks induction but not expression of LTP. Science 245:862-866.

Miller SG, Kennedy MB (1985) Distinct forebrain and cerebellar isozymes of type II $\mathrm{Ca}^{2+} /$ calmodulin-dependent protein kinase associate differently with the postsynaptic density fraction. J Biol Chem 260:9039-9046.

Petralia RS, Wenthold RJ (1992) Light and electron immunocytochemical localization of AMPA-selective glutamate receptors in the rat brain. J Comp Neurol 318:329-354.

Pettit DL, Perlman S, Malinow R (1994) Potentiated transmission and prevention of further LTP by increased CaMKII activity in postsynaptic hippocampal slice neurons. Science 246:1881-1885.

Pettit DL, Koothan T, Liao D, Malinow R (1995) Vaccinia virus transfection of hippocampal slice neurons. Neuron 14:685-688.
Rubio ME, Wenthold RJ (1997) Glutamate receptors are selectively targeted to postsynaptic sites in neurons. Neuron 18:939-950.

Scholz WK, Baitinger C, Schulman H, Kelly PT (1988) Developmental changes in $\mathrm{Ca}^{2+} /$ calmodulin-dependent protein kinase II in cultures of hippocampal pyramidal neurons and astrocytes. J Neurosci 8:1039-1051.

Schweitzer ES, Sanderson MJ, Wasterlain CG (1995) Inhibition of regulated catecholamine secretion from PC12 cells by the $\mathrm{Ca}^{2+} /$ calmodulin kinase II inhibitor KN-62. J Cell Sci 108:2619-2628.

Shirke AM, Malinow R (1997) Mechanisms of potentiation by calciumcalmodulin kinase II of postsynaptic sensitivity in rat hippocampal CA1 neurons. J Neurophysiol 78:2682-2692.

Silva AJ, Stevens CF, Tonegawa S, Wang Y (1992) Deficient hippocampal long-term potentiation in $\alpha$-calcium-calmodulin kinase II mutant mice. Science 257:201-206.

Spacek J, Harris KM (1997) Three-dimensional organization of smooth endoplasmic reticulum in hippocampal CA1 dendrites and dendritic spines of the immature and mature rat. J Neurosci 17:190-203.

Steinhardt RA, Bi G, Alderton JM (1994) Cell membrane resealing by a vesicular mechanism similar to neurotransmitter release. Science 263:390-393.

Tighilet B, Hashikawa T, Jones EG (1998) Cell- and lamina-specific expression and activity-dependent regulation of type II calcium/ calmodulin-dependent protein kinase isoforms in monkey visual cortex. J Neurosci 18:2129-2146.

Tokumitsu H, Chijiwa T, Hagiwara M, Mizutani A, Terasawa M, Hidaka $\mathrm{H}$ (1990) KN-62, 1-[N,O-bis (5-isoquinolinesulfonyl) $-N$-methyl-Ltyrosyl]-4-phenylpiperazine, a specific inhibitor of $\mathrm{Ca}^{2+} /$ calmodulindependent protein kinase II. J Biol Chem 265:4315-4320.

Waxham MN, Malenka R, Kelly PT, Mauk MD (1993) Calcium/ calmodulin-dependent protein kinase II regulates hippocampal synaptic transmission. Brain Res 609:1-8.

Wu G, Malinow R, Cline HT (1996) Maturation of a central glutamatergic synapse. Science 274:972-976. 